\title{
Mandibular Ridge Splitting and Gradual Bone Expansion Technique for Immediate Placement of Implant in the Posterior Thin Region: A Clinical Report
}

\author{
Massoumeh Khoshhal ${ }^{1}$; Parviz Torkzaban ${ }^{1}$; Fariborz Vafaee ${ }^{2}$; Shilan Razaghi ${ }^{1,}$ \\ ${ }_{1}^{1}$ Department of Periodontics, School of Dentistry, Hamadan University of Medical Sciences, Hamadan, IR Iran \\ ${ }^{2}$ Department of Prosthodontics, School of Dentistry, Hamadan University of Medical Sciences, Hamadan, IR Iran \\ ${ }^{*}$ Corresponding author: Shilan Razaghi, Department of Periodontics, School of Dentistry, Hamadan University of Medical Sciences, Hamadan, IR Iran. Tel: +98-8118381085, Fax: +98- \\ 8118381086, E-mail: shilan.r@gmail.com
}

Received: May 2, 2013; Revised: June 29, 2013; Accepted: June 29, 2013

\begin{abstract}
Introduction: Narrow alveolar ridges especially in posterior mandibular remains a serious challenge for successful placement of endosseous implants.

Case Presentation: This case report addresses surgical procedures for widening the atrophic ridge by means of splitting the crest of an edentulous ridge as thin as $2.5 \mathrm{~mm}$ and gradual expansion in the posterior mandibular ridge, then simultaneous placement of dental implants within the split ridge. A significant increase was achieved in the bone dimension, which enabled the placement of endosseous dental implants successfully.

Discussion: This segmental ridge-split procedure with gradual bone expansion provides a quicker method wherein an atrophic ridge can be predictably expanded and eliminating the need for a second surgical site. This technique also shows that immediate implantation in split ridge of mandible can be performed.
\end{abstract}

Keywords:Dental Implants; Alveolar Bone Loss; Osteotomy

\section{Introduction}

At present, patients are more interested in dental treatments with better esthetic results and less treatment time. Rehabilitation of occlusion with dental implants is considered one of the most efficient treatment methods for edentulism (1). Lack of sufficient bone to place an implant at the functionally and aesthetically most appropriate position is a common problem. This happens after the extraction of teeth if the patient has been missing teeth for a considerable period of time. Treatment of atrophic ridge especially in posterior mandibular is accompanied with great problem in achieving successful results with endosseous implants. Although different techniques exist for reconstruction of atrophic ridge, there are chances of surgical risk, postoperative morbidity and multiple surgeries $(2,3)$. Various surgical widening techniques have been described, including lateral augmentation with $(4,5)$ or without guided bone regeneration (GBR) (6), ridge expansion osteotomy $(7,8)$, ridge splitting technique with $(9,10)$ or without (11) interpositional grafting and horizontal distraction osteogenesis (12). According to Atwood (13) knife-edge crests might be managed by conventional bone grafting, guided bone augmentation procedures by using membranes, and various other techniques. Ridge augmentation by bone graft requires a second surgery for a later implantation, thus lengthening the treatment time and cost. Ridge splitting technique which causes lateral ridge expansion creates new implant bed by longitudinal osteotomy positioning buccal cortex laterally (14). The buccal cortex is positioned laterally to create space between buccal and lingual cortical plates, which is filled by endosseous implant with or without any graft material $(15,16)$. This technique is performed with immediate implant placement, which decreases the treatment time significantly. Other advantages include lesser overall cost, no need of barrier membranes or bone graft materials and no morbidity related to second donor site. Although, this technique is more suitable for maxilla and can be performed in posterior mandibular region if favorable condition exists (15). Favorable conditions of posterior mandible for ridge splitting techniques include long edentulous span, abundant bone height and presence of cancellous bone between the dense outer cortical plates $(16,17)$. A staged approach to ridge splitting in the mandible can be performed to avoid complications. Another technique for placement of dental implants in narrow bone ridges is repositioning and remodeling of alveolar bone by controlled expansion. This technique uses screwtype configuration osteotomes and threadformers with

Copyright (C) 2013, Hamadan University of Medical Sciences. This is an open-access article distributed under the terms of the Creative Commons Attribution License, which permits unrestricted use, distribution, and reproduction in any medium, provided the original work is properly cited. 
Khoshhal M et al.

increasing diameters (18). This clinical report describes the technique for ridge splitting, gradual expansion in the mandible and simultaneous implant placement within the split ridge.

\section{Case Presentation}

The patient was a 35-year-old female. Her chief complaint was missing teeth number 19 and 20 in lower left region of the jaw. She requested fixed prosthesis, preferably an implant-supported. Her expectations were reasonable. Her medical history was noncontributory. Extraand intraoral examinations had normal findings, and her dentition was in a good state of repair. Dental history revealed missing mandibular left teeth number 19 and 20 , which had been extracted five years ago. Cone beam computed tomography was performed to evaluate the bone quality and quantity. CT scan revealed inadequate buccolingual dimension of bone at the crest for implant placement. There was adequate cortical and cancellous bone to allow ridge expansion. It was decided to place immediate implants, using the split control expanding technique. Antiinflammatory drugs were prescribed for patient before and after the implantation. After local anesthesia, a remote incision to preservation keratinized tissue and intracrevicular incisions were made around buccal aspect of adjacent teeth. Full thickness mucoperiosteal flaps were raised on the buccal and lingual aspects of cortical plates but minimal tissue reflection was performed in lingual aspect to preserve the periosteum attachment surrounding the buccal and lingual bone. This was performed to prevent possible buccal bone plate crack. Keeping the periosteum intact would facilitate repositioning of the fragments and achieve good healing. With a surgical guide, the exact position for the implant was scored. Corticotomy was performed with a scalpel (No.15) to use as autogenous graft and facilitating the osteotomy of region. The horizontal osteotomy line was cut along the narrow crest using a thin separating disk (6.66 $\mathrm{mm}), 1$ to $2 \mathrm{~mm}$ away from the second molar till the first premolar region on the left side of the mandible under saline irrigation (Figure 1). Then the osteotomy line was deepened with wider disk $(9.50 \mathrm{~mm})$. A sequence of expansion drill of increasing width in the two select sites was used to allow more gradual bone expansion (Figure 1). Then the implant sites were prepared using final twist drills and implants of $3.4 \mathrm{~mm} \times 10 \mathrm{~mm}, 4.5 \mathrm{~mm} \times 10 \mathrm{~mm}$ were placed in premolar and molar regions. The cover screws were placed and autogenous cortical bone-graft with allograft material was used to fill the buccal aspect of region (Figure 2). Then tension free mucoperiosteal tissue closure was performed over implants using 3-0 nonresorbable suture. Nonsteroidal analgesics, Amoxicillin $500 \mathrm{mg}$ and $0.2 \%$ chlorhexidine mouth rinse was the preoperative protocol administered for the patient. Sutures were removed after 10 days.
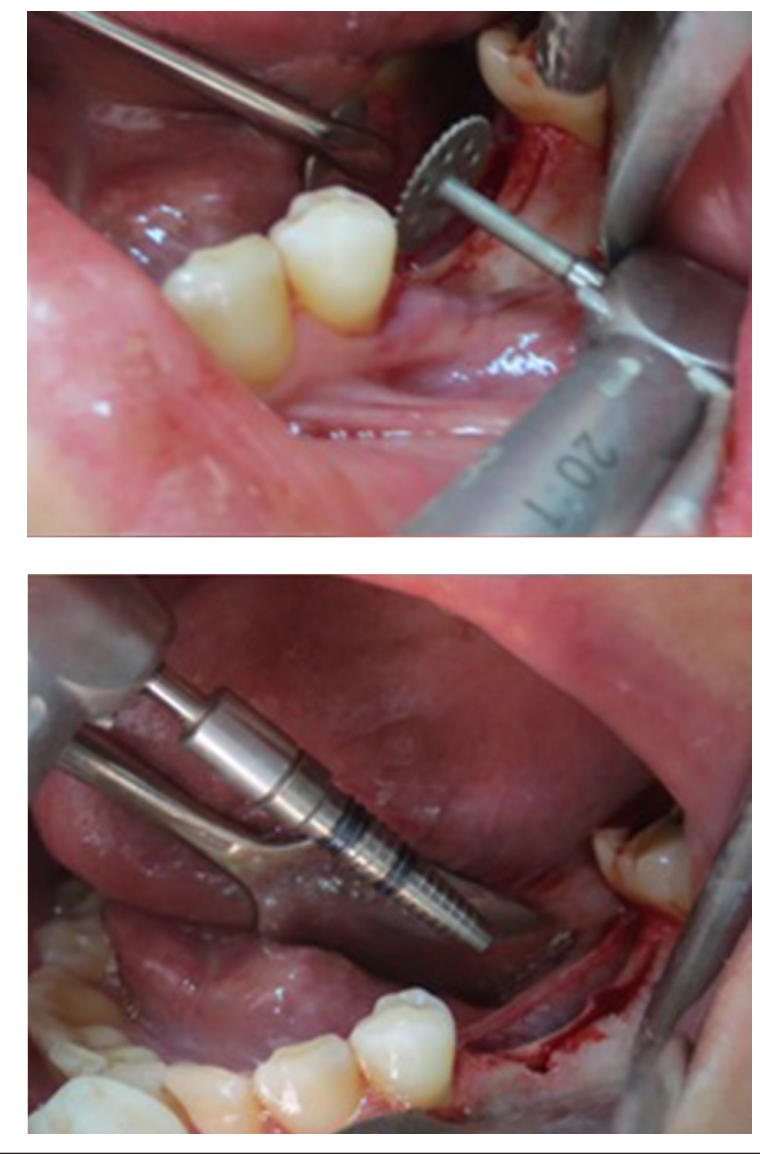

Figure 1. Initial Osteotomy With Separating Disk and Expansion Drill Being Used for Final Osteotomy and Implant Site Preparation
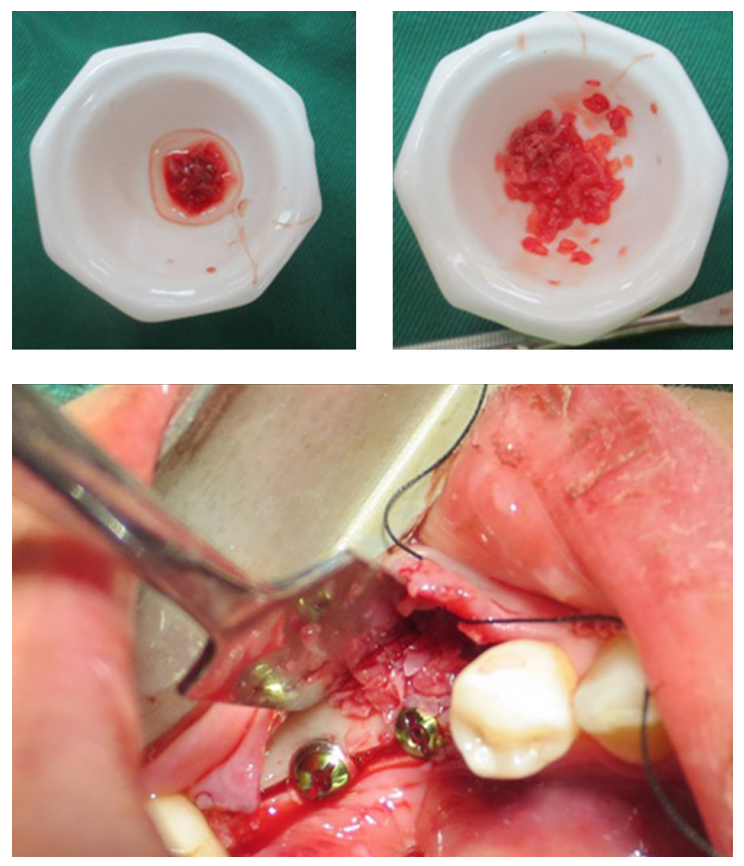

Figure 2. Two Implants Were Placed and a Mixture of Autogenous and Allograft Material Were Used 


\section{Khoshhal M et al.}

A significant increase was achieved in the bone dimension, which enabled the placement of endosseous dental implants successfully. Then after preparation of the implant sites, implants of $3.4 \mathrm{~mm} \times 10 \mathrm{~mm}, 4.5 \mathrm{~mm} \times 10 \mathrm{~mm}$ were placed in premolar and molar regions. This report demonstrated the successful use of expanding the posterior mandibular alveolar ridge. It also showed that this technique allows for immediate implant placement.

\section{Discussion}

The posterior mandible is the most difficult region for reconstruction and early implantation in cases of severe alveolar resorption in the maxillomandibular complex. Onlay grafting with biodegradable membranes and autografts is the most frequently used technique; however, this technique involves a long ossification period, and the tendency of the graft material to resorb can easily decrease bone quality and quantity (19). Time lost and donor-side morbidity are the main disadvantages of this reconstructive approach. The split-crest technique should be delineated as a bone expansion procedure, which potentially eliminates the overall disadvantages of Onlay grafting for esthetic and functional demands. Chiapasco et al. evaluated the efficiency of different surgical techniques for ridge reconstruction and success rates of implants placed in the augmented areas. The surgical success and the implant survival rates were as high as the guided bone regeneration and Onlay graft procedure (4), with the advantage of a shorter treatment time. Careful preparation of the bone and maintenance of an attached periosteum are critical to the formation of new bone around the interproximal surfaces of the implants. Wound healing in these cases is similar to the fracture repair of bone. The gap is filled with a blood clot, which is organized and replaced with woven bone and further matures into load-bearing lamellar bone at the implant interface (19). The ridge splitting technique is used to expand the edentulous ridge for implantation or insertion of interpositional bone graft (15). Significant advantages of ridge expansion rather than Onlay grafting include simultaneous implant placement and grafting, lower cost, lower possibility of cross-infection from graft materials and lower morbidity. This technique has greater predictability, since the grafted area is essentially a five-wall bony defect, with excellent blood supply. This technique is only suitable for enhancing ridge width. There must be adequate available bone height for implant placement, and no vertical bone defect should be present. A minimum of $3 \mathrm{~mm}$ of bone width, including at least $1 \mathrm{~mm}$ of cancellous bone is desired to insert a bone chisel between cortical plates and consequently expanding the cortical bones. The thinner cortical plates and softer medullary bone make the maxillary ridge easier to expand. The risk of malfracture of the osteotomized segment is high in the mandible due to thicker cortical plates (20). Favorable conditions for the posterior man- dible include a long edentulous span (missing molar and premolar teeth), abundant bone height superior to the mandibular canal (>12 mm), and presence of some cancellous bone between the dense outer cortical plates. The disadvantage of this procedure is that if the complication arises and bone loss occurs ,the patient is left with a larger bone defect than before (16). If favorable conditions are not present, clinician might prefer Onlay augmentation. Therefore, appropriate case selection and surgical technique is of great importance when considering the application of this technique.

\section{References}

1. Zarb GA, Schmitt A. The longitudinal clinical effectiveness of osseointegrated dental implants: the Toronto Study. Part II: The prosthetic results. J Prosthet Dent. 1990;64(1):53-61.

2. Garg AK, Morales MJ, Navarro I, Duarte F. Autogenous mandibular bone grafts in the treatment of the resorbed maxillary anterior alveolar ridge: rationale and approach. Implant Dent. 1998;7(3):169-76.

3. Buser D, Dula K, Lang NP, Nyman S. Long-term stability of osseointegrated implants in bone regenerated with the membrane technique. 5-year results of a prospective study with 12 implants. Clin Oral Implants Res. 1996;7(2):175-83.

4. Chiapasco M, Abati S, Romeo E, Vogel G. Clinical outcome of autogenous bone blocks or guided bone regeneration with e-PTFE membranes for the reconstruction of narrow edentulous ridges. Clin Oral Implants Res. 1999;10(4):278-88.

5. De Boever AL, De Boever JA. A one-stage approach for nonsubmerged implants using a xenograft in narrow ridges: report on seven cases. Int J Periodontics Restorative Dent. 2003;23(2):169-75.

6. Bedrossian E, Tawfilis A, Alijanian A. Veneer grafting: a technique for augmentation of the resorbed alveolus prior to implant placement. A clinical report. Int J Oral Maxillofac Implants. 2000;15(6):853-8.

7. Summers RB. The osteotome technique: Part 2--The ridge expansion osteotomy (REO) procedure. Compendium. 1994;15(4):422424, 426, passim. quiz 436.

8. Sethi A, Kaus T. Maxillary ridge expansion with simultaneous implant placement: 5-year results of an ongoing clinical study. Int J Oral Maxillofac Implants. 2000;15(4):491-9.

9. Lustmann J, Lewinstein I. Interpositional bone grafting technique to widen narrow maxillary ridge. Int J Oral Maxillofac Implants. 1995;10(5):568-77.

10. Engelke WG, Diederichs CG, Jacobs HG, Deckwer I. Alveolar reconstruction with splitting osteotomy and microfixation of implants. Int J Oral Maxillofac Implants. 1997;12(3):310-8.

11. Simion M, Baldoni M, Zaffe D. Jawbone enlargement using immediate implant placement associated with a split-crest technique and guided tissue regeneration. Int J Periodontics Restorative Dent. 1992;12(6):462-73.

12. Takahashi T, Funaki K, Shintani H, Haruoka T. Use of horizontal alveolar distraction osteogenesis for implant placement in a narrow alveolar ridge: a case report. Int J Oral Maxillofac Implants. 2004;19(2):291-4.

13. Atwood DA. Some clinical factors related to rate of resorption of residual ridges. 1962. J Prosthet Dent. 2001;86(2):119-25.

14. Enislidis G, Wittwer G, Ewers R. Preliminary report on a staged ridge splitting technique for implant placement in the mandible: a technical note. Int J Oral Maxillofac Implants. 2006;21(3):445-9.

15. Basa S, Varol A, Turker N. Alternative bone expansion technique for immediate placement of implants in the edentulous posterior mandibular ridge: a clinical report. Int J Oral Maxillofac Implants. 2004;19(4):554-8.

16. Misch CM. Implant site development using ridge splitting techniques. Oral Maxillofac Surg Clin North Am. 2004;16(1):65-74.

17. Misch CM. Ridge augmentation using mandibular ramus bone grafts for the placement of dental implants: presentation of a 


\section{Khoshhal M et al.}

technique. Pract Periodontics Aesthet Dent.1996;8(2):127-35. quiz 138.

18. Misch CM. Use of the mandibular ramus as a donor site for onlay bone grafting. J Oral Implantol. 2000;26(1):42-9.

19. Ignatius AA, Ohnmacht M, Claes LE, Kreidler J, Palm F. A composite polymer/tricalcium phosphate membrane for guided bone regeneration in maxillofacial surgery. J Biomed Mater Res. 2001;58(5):564-9.

20. Hollinger J, Wong ME. The integrated processes of hard tissue regeneration with special emphasis on fracture healing. Oral Surg Oral Med Oral Pathol Oral Radiol Endod.1996;82(6):594-606. 